\title{
Increased abundance of Sutterella spp. and Ruminococcus torques in feces of children with autism spectrum disorder
}

\author{
Lv Wang ${ }^{1}$, Claus T Christophersen², Michael J Sorich', Jacobus P Gerber ${ }^{1}$, Manya T Angley ${ }^{1}$ \\ and Michael A Conlon ${ }^{2 *}$
}

\begin{abstract}
Background: A recent report indicated that numbers of Sutterella spp. are elevated in gastrointestinal biopsies taken from children with autism spectrum disorder (ASD). We have recently reported changes in the numbers of some bacteria within the stool of ASD children, and now examine whether numbers of Sutterella spp. and some other mucosa-associated bacteria linked with gastrointestinal disease (Ruminococcus gnavus and Ruminococcus torques) are also altered in the stool of these children.
\end{abstract}

Findings: We show that numbers of Sutterella spp. are elevated in feces of ASD children relative to controls, and that numbers of $R$. torques are higher in the children with ASD with a reported functional gastrointestinal disorder than those without such a disorder.

Conclusions: We show further evidence of changes in the gut microbiota of children with ASD and confirm that the abundance of Sutterella spp. is altered in stool.

Keywords: Autism spectrum disorder, Gut, Feces, Microbiota, Sutterella

\section{Findings}

Autism spectrum disorder (ASD) is a neurodevelopmental disorder where there is evidence of gastrointestinal (GI) disturbance in many affected individuals. Several studies have demonstrated an altered GI microbiota in children with ASD compared with controls [1-4]. Recently, Williams et al. [5] reported a significantly higher prevalence of Sutterella spp. in biopsies taken from the GI tract of ASD children with GI disturbance compared to controls with GI disturbance. Bacteria of the genus Sutterella have been identified in canine and human feces [6,7]. Sutterella wadsworthensis is noteworthy as it has been associated with some GI infections in humans [8]. In one study using culture-based techniques, less than $1 \%$ of individuals had detectable fecal levels of $S$. wadsworthensis, even among individuals with bowel disorders [9]. However, in another study, where it was shown that the

\footnotetext{
* Correspondence: michael.conlon@csiro.au

${ }^{2}$ Preventative Health National Research Flagship, CSIRO Animal, Food and Health Sciences, Gate 13, Kintore Avenue, Adelaide, South Australia 5001, Australia

Full list of author information is available at the end of the article
}

abundance of $S$. wadsworthensis in colonic biopsies of adults with inflammatory bowel disease (IBD) was not different from controls, Sutterella appeared to be more prevalent in the human gut than previously reported [10].

A previous study by our group used quantitative real-time PCR (QPCR) to compare the abundance of a range of bacteria in feces of children with ASD, their siblings and community controls, and demonstrated a low relative abundance of Bifidobacterium spp. and the mucolytic bacterium Akkermansia muciniphila in children with ASD [11]. We have carried out further QPCR analyses of samples from that study to examine whether the abundance of Sutterella spp. is also altered in the feces of children with ASD relative to controls. We have also examined whether fecal numbers of some other mucosa-associated bacteria, namely Ruminococcus torques and Ruminococcus gnavus, are altered in individuals with ASD. As with A. muciniphila, mucosal numbers of Ruminococcus species are altered in individuals with IBD [12].

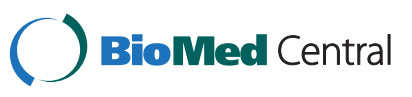

(c) 2013 Wang et al.; licensee BioMed Central Ltd. This is an open access article distributed under the terms of the Creative Commons Attribution License (http://creativecommons.org/licenses/by/2.0), which permits unrestricted use, distribution, and reproduction in any medium, provided the original work is properly cited. 
Fecal samples were collected from 23 children with ASD (3 without siblings), 22 typically developing siblings (SIB) and 9 community controls (CON) without a family history of ASD. The study complied with National Health and Medical Research Council (Australia) guidelines relating to ethical conduct in human research. Participants' caregivers completed a functional GI disorder (FGID) questionnaire [11]. Procedures for fecal sample collection and processing, as well as microbial DNA extraction, have been described previously [11]. QPCR was used to estimate the abundance of Sutterella spp., R. torques and $R$. gnavus in feces using previously described primers $[5,12]$. Methods for QPCR, data analysis and primers for total bacteria were also as reported previously [11]. For Sutterella, SsoFast ${ }^{\mathrm{TM}}$ Probes Supermix was used instead of EvaGreen ${ }^{\circ}$ Supermix and an updated version of Bio-Rad CFX Manager software (Version 2.0) was used. Statistical analyses were carried out using SPSS for Windows ${ }^{\text {TM }}$ (version 17, SPSS Inc., Chicago, IL, USA). Analyses were carried out using analysis of variance (ANOVA) followed by Dunnett's test or by Student's $t$-test. A $P$ value of less than 0.05 was considered statistically significant.

The absolute numbers (per gram of feces) and relative numbers of bacteria in feces of individuals from the ASD, SIB and CON groups are presented in Table 1. Sutterella was detected in the majority of samples analyzed (ASD 21/23; SIB 19/22; CON 9/9). ANOVA revealed significantly different absolute and relative numbers of Sutterella in ASD children compared with sibling and community controls ( $P=0.044$ and $P=0.05$, respectively). A post hoc analysis showed significantly increased absolute and relative numbers of Sutterella in individuals with ASD compared with community controls $(P=0.028$ and $P=0.03$, respectively). Moreover, absolute fecal numbers of Sutterella tended to be higher in siblings than community controls $(P=0.047) . R$. torques and
R. gnavus were detected in all fecal samples. Absolute numbers of $R$. torques were not significantly higher in ASD children than in other groups (ANOVA, $P=0.08$ ) but were higher in siblings relative to community controls $(P=0.046)$. Absolute and relative numbers of $R$. gnavus did not differ significantly between groups. Significantly elevated absolute numbers of $R$. torques were evident in children with ASD whose caregivers reported them having a FGID (nine individuals) compared to those without reported FGID (14 individuals) (Student's $t$-test, $P=0.008$ ). There was no significant difference in fecal numbers of Sutterella or R. gnavus between ASD children with and without reported FGIDs (data not shown).

Our results demonstrate that numbers of Sutterella are elevated in the feces of children with ASD relative to community controls. This confirms and builds on the findings of Williams et al. [5], who showed high detection rates of these bacteria in GI biopsies taken from ASD children with a GI disturbance. In our study there was no evidence of a difference in numbers of Sutterella between ASD children with or without caregiver-reported FGID. It is not yet evident what the consequences of an increase in fecal Sutterella populations means but it is possible that under specific conditions these bacteria could cause infection. Our findings add to the growing list of bacterial groups whose abundance appears to be altered in the GI tract of children with ASD. Our results also indicate that fecal samples may suffice for the detection and quantification of Sutterella in the human gut, including children with ASD. This contrasts with a previous estimation of detectability and abundance in human fecal samples by Engberg et al. [9]. Previously it has been difficult to culture and isolate anaerobic and microaerophilic bacteria, such as Sutterella, using traditional microbiological techniques and it is likely the QPCR method we employed was more sensitive than the culture method used by the

Table 1 Numbers of targeted bacteria in feces of children with ASD, their siblings and community controls

\begin{tabular}{|c|c|c|c|}
\hline \multirow[t]{2}{*}{ Bacterial targets } & \multicolumn{3}{|c|}{ Bacterial number (mean \pm standard error of the mean) } \\
\hline & Autism spectrum disorder $(n=23)$ & Siblings $(n=22)$ & Community controls $(n=9)$ \\
\hline \multicolumn{4}{|c|}{ Absolute number per gram feces } \\
\hline Sutterella spp. & $(1.1 \pm 0.4) \times 10^{6^{*}}$ & $(8.7 \pm 2.5) \times 10^{5^{*}}$ & $(6.7 \pm 4.5) \times 10^{5}$ \\
\hline R. torques & $(3.5 \pm 1.6) \times 10^{5}$ & $(4.2 \pm 1.5) \times 10^{5^{*}}$ & $(3.5 \pm 1.4) \times 10^{4}$ \\
\hline R. gnavus & $(4.5 \pm 0.7) \times 10^{5}$ & $(5.1 \pm 0.9) \times 10^{5}$ & $(2.9 \pm 0.4) \times 10^{5}$ \\
\hline Total bacteria & $(1.2 \pm 0.2) \times 10^{9}$ & $(1.4 \pm 0.3) \times 10^{9}$ & $(1.3 \pm 0.2) \times 10^{9}$ \\
\hline \multicolumn{4}{|l|}{ Relative numbers ${ }^{2}$} \\
\hline Sutterella spp. & $3.0 \pm 0.7^{*}$ & $7.4 \pm 3.5^{*}$ & $0.5 \pm 0.3$ \\
\hline R. torques & $4.8 \pm 1.8$ & $4.8 \pm 1.2$ & $1.1 \pm 0.2$ \\
\hline R. gnavus & $1.3 \pm 0.2$ & $1.8 \pm 0.5$ & $0.6 \pm 0.2$ \\
\hline
\end{tabular}

${ }^{1}$ Means with an asterisk differ from those of community controls. $P<0.05$ (one-way ANOVA followed by Dunnett's test).

${ }^{2}$ Relative numbers were calculated using qBase+ $[13,14]$.

$A S D$ autism spectrum disorder. 
previous study [9]. However, our detection rates of Sutterella in humans are comparable with detection rates in colonic biopsies from human adults [10].

In this study we also extended our analysis of mucusdegrading bacteria in feces of ASD children, having previously shown a low abundance of the mucolytic bacterium $A$. muciniphila. We measured the abundances of $R$. torques and $R$. gnavus, both of which can degrade mucus $[15,16]$ and have been associated with GI disturbance [17]. We have shown there is a trend of increased fecal numbers of $R$. torques in ASD children. This pattern of decreased numbers of $A$. muciniphila but increased numbers of $R$. torques in feces of ASD children is congruent with the pattern observed in the mucosa of adults with IBD [12]. In the latter study it was also found that growth of $A$. muciniphila was inhibited by co-culture with MUC2, the predominant form of mucin in the large bowel, whereas this mucin augmented the growth of other bacteria such as $R$. torques. Although it is likely that the populations and activities of microbes that adhere to the mucus lining the large bowel may differ substantially from those in the feces, it is also likely that some changes in gut mucosal microbial populations are reflected in, and hence detectable in, feces. Changes in the amount of mucus produced may drive changes in mucus-degrading microbes in both the mucosa and in feces, as mucus can be incorporated into the fecal stream. Individuals with IBD or ASD may have changes in large bowel mucus production that could impact (or are a result of changes in) the mucosal barrier of the gut. Indeed, increased gut permeability has been reported in a subgroup of children with ASD $[18,19]$. Further studies that look at gut mucus production and turnover in children with ASD are warranted.

In summary, we show further evidence of changes in the gut microbiota of children with ASD, now demonstrating that fecal abundances of Sutterella and $R$. torques are altered (the latter in those reported to have FGID). The role that these particular bacteria play in ASD is not yet understood but they may simply reflect larger scale shifts in the microbial populations as a result of the condition.

\section{Abbreviations}

ANOVA: Analysis of variance; ASD: Autism spectrum disorder; CON: Control group; FGID: Functional gastrointestinal disorder; Gl: Gastrointestinal; IBD: Inflammatory bowel disease; QPCR: Quantitative real-time polymerase chain reaction; SIB: Sibling group.

\section{Competing interests}

The authors declare that they have no competing interests.

\section{Authors' contributions}

CTC, JPG, LW, MAC, MJS and MTA contributed to the design and planning of the study. LW conducted the experiment and analytical methods. CTC, LW and MAC contributed to the analysis of the results, LW, MAC and MTA prepared the manuscript, and all authors read and approved the manuscript.

\section{Acknowledgments}

This research was supported by the Australian Rotary Health Research Fund from Australian Rotary Health. We wish to express our gratitude to the participating children and their parents. We would also like to acknowledge Dr Richard Couper, pediatric gastroenterologist, for medical advice, and Emma Watson, Michelle Vuaran and Jennifer Giles for technical assistance. The authors have no conflicts of interest to declare.

\section{Author details}

'Sansom Institute for Health Research, University of South Australia, GPO Box 2471, Adelaide, South Australia 5001, Australia. ${ }^{2}$ Preventative Health National Research Flagship, CSIRO Animal, Food and Health Sciences, Gate 13, Kintore Avenue, Adelaide, South Australia 5001, Australia.

Received: 20 June 2013 Accepted: 25 September 2013 Published: 4 November 2013

\section{References}

1. Finegold SM, Dowd SE, Gontcharova V, Liu C, Henley KE, Wolcott RD, Youn E, Summanen PH, Granpeesheh D, Dixon D, Liu M, Molitoris DR, Green JA: Pyrosequencing study of fecal microflora of autistic and control children. Anaerobe 2010, 16:444-453.

2. Williams BL, Hornig M, Buie T, Bauman ML, Cho Paik M, Wick I, Bennett A, Jabado O, Hirschberg DL, Lipkin WI: Impaired carbohydrate digestion and transport and mucosal dysbiosis in the intestines of children with autism and gastrointestinal disturbances. PLOS One 2011, 6:e24585.

3. Parracho HM, Bingham MO, Gibson GR, McCartney AL: Differences between the gut microflora of children with autistic spectrum disorders and that of healthy children. J Med Microbiol 2005, 54:987-991.

4. Kang DW, Park JG, Ilhan ZE, Wallstrom G, Labaer J, Adams JB, KrajmalnikBrown R: Reduced incidence of Prevotella and other fermenters in intestinal microflora of autistic children. PLoS One 2013, 8:e68322.

5. Williams BL, Hornig M, Parekh T, Lipkin Wl: Application of novel PCR-based methods for detection, quantitation, and phylogenetic characterization of Sutterella species in intestinal biopsy samples from children with autism and gastrointestinal disturbances. mBio 2012, 3:1-11.

6. Sakon H, Nagai F, Morotomi M, Tanaka R: Sutterella parvirubra sp. nov. and Megamonas funiformis sp. nov., isolated from human faeces. Int I Syst Evol Microbiol 2008, 58:970-975.

7. Greetham HL, Collins MD, Gibson GR, Giffard C, Falsen E, Lawson PA: Sutterella stercoricanis sp. nov., isolated from canine faeces. Int I Syst Evol Microbiol 2004, 54:1581-1584.

8. Molitoris E, Wexler HM, Finegold SM: Sources and antimicrobial susceptibilities of Campylobacter gracilis and Sutterella wadsworthensis. Clin Infect Dis 1997, 25(Suppl 2):S264-S265.

9. Engberg J, On SL, Harrington CS, Gerner-Smidt P: Prevalence of campylobacter, arcobacter, helicobacter, and Sutterella spp. in human fecal samples as estimated by a reevaluation of isolation methods for campylobacters. J Clin Microbiol 2000, 38:286-291.

10. Mukhopadhya I, Hansen R, Nicholl CE, Alhaidan YA, Thomson JM, Berry SH, Pattinson C, Stead DA, Russell RK, El-Omar EM, Hold GL: A comprehensive evaluation of colonic mucosal isolates of Sutterella wadsworthensis from inflammatory bowel disease. PLoS One 2011, 6:e27076.

11. Wang L, Christophersen CT, Sorich MJ, Gerber JP, Angley MT, Conlon MA: Low relative abundances of the mucolytic bacterium Akkermansia muciniphila and Bifidobacterium spp. in feces of children with autism. Appl Environ Microbiol 2011, 77:6718-6721.

12. Png CW, Lindén SK, Gilshenan KS, Zoetendal EG, McSweeney CS, Sly LI, McGuckin MA, Florin THJ: Mucolytic bacteria with increased prevalence in IBD mucosa augment in vitro utilization of mucin by other bacteria. Am J Gastroenterol 2010, 105:2420-2428.

13. Hellemans J, Mortier G, De Paepe A, Speleman F, Vandesompele J: qBase relative quantification framework and software for management and automated analysis of real-time quantitative PCR data. Genome Biol 2007, 8:R19.

14. Vandesompele J, De Preter K, Pattyn F, Poppe B, Van Roy N, De Paepe A Speleman F: Accurate normalization of real-time quantitative RT-PCR data by geometric averaging of multiple internal control genes. Genome Biol 2002, 3:RESEARCH0034.

15. Hoskins LC, Agustines M, McKee WB, Boulding ET, Kriaris M, Niedermeyer G: Mucin degradation in human colon ecosystems. Isolation and properties 
of fecal strains that degrade $\mathrm{ABH}$ blood group antigens and oligosaccharides from mucin glycoproteins. J Clin Invest 1985, 75:944-953.

16. Dethlefsen L, Eckburg PB, Bik EM, Relman DA: Assembly of the human intestinal microbiota. Trends Ecol Evol 2006, 21:517-523.

17. Joossens M, Huys G, Cnockaert M, De Preter V, Verbeke K, Rutgeerts $P$, Vandamme $P$, Vermeire $S$ : Dysbiosis of the faecal microbiota in patients with Crohn's disease and their unaffected relatives. Gut 2011, 60:631-637.

18. de Magistris L, Familiari V, Pascotto A, Sapone A, Frolli A, lardino P, Carteni M, De Rosa M, Francavilla R, Riegler G, Militerni R, Bravaccio C: Alterations of the intestinal barrier in patients with autism spectrum disorders and in their first-degree relatives. J Pediatr Gastr Nutr 2010, 51:418-424.

19. D'Eufemia P, Celli M, Finocchiaro R, Pacifico L, Viozzi L, Zaccagnini M, Cardi E, Giardini O: Abnormal intestinal permeability in children with autism. Acta Paediatr 1996, 85:1076-1079.

doi:10.1186/2040-2392-4-42

Cite this article as: Wang et al:: Increased abundance of Sutterella spp. and Ruminococcus torques in feces of children with autism spectrum disorder. Molecular Autism 2013 4:42.

\section{Submit your next manuscript to BioMed Central and take full advantage of:}

- Convenient online submission

- Thorough peer review

- No space constraints or color figure charges

- Immediate publication on acceptance

- Inclusion in PubMed, CAS, Scopus and Google Scholar

- Research which is freely available for redistribution 